\title{
ZINC4085554 inhibits cancer cell adhesion by interfering with the interaction of Akt1 and FAK
}

\author{
SHYAM K. MORE, EMILIE E. VOMHOF-DEKREY and MARC D. BASSON \\ Department of Surgery, University of North Dakota School of Medicine and Health Sciences, \\ Grand Forks, ND 58202, USA
}

Received October 18, 2018; Accepted February 27, 2019

DOI: $10.3892 / \mathrm{ol} .2019 .10192$

\begin{abstract}
Perioperative or circulatory forces enhance disseminated cancer cell adhesiveness by modulating focal adhesion kinase (FAK)-Akt1 interaction. Selectively blocking FAK-Akt1 interaction by a peptide derived from the FAK-Four-point-one, ezrin, radixin, moesin (FERM) domain reduces colon cancer cell adhesion in vitro and in mice. A preliminary in silico screening identified two small molecules resembling a peptide that may inhibit pressure-stimulated SW620 cancer cell adhesion to collagen I. The present study selected ZINC4085554 for further study to validate its proposed mechanism of action, using human SW620 colon cancer cells as a model system. At 25 and $50 \mu \mathrm{M}$, ZINC4085554 inhibited the pressure-stimulated adhesion of SW620 colon cancer cells to collagen I. This molecule prevented pressure-stimulated FAK-Tyr-397 phosphorylation; however, it did not affect Akt1-Ser-473 phosphorylation, indicating that ZINC4085554 acts downstream of Akt1, while Akt-Thr-308 remains unchanged in the presence of pressure and or ZINC4085554. Indeed, ZINC4085554 inhibited FAK-Akt1 interaction in response to increased extracellular pressure, consistent with the proposed mechanism. ZINC4085554 did not inhibit FAK-Tyr-397 phosphorylation in response to cell adhesion to collagen I, indicating the specificity of the inhibitory effects towards force-stimulated pathways. Finally, the present study confirmed that ZINC4085554 at $50 \mu \mathrm{M}$ prevented pressure-activation of adhesion to surgical wounds in vivo in parallel to its ablation of intracellular signaling. In summary, ZINC4085554 is a small molecule mimicking part of the structure of FAK that reduces cancer cell adhesion by impairing pressure-stimulated FAK-Akt1 interaction and its downstream consequences. ZINC4085554 does not inhibit conventional outside-in FAK signaling and may be less toxic
\end{abstract}

Correspondence to: Dr Marc D. Basson, Department of Surgery, University of North Dakota School of Medicine and Health Sciences, 1301 North Columbia Road, Grand Forks, ND 58202, USA

E-mail:marc.basson@und.edu

Key words: adhesion, pressure, small molecule, focal adhesion kinase, Akt1, colon cancer, metastasis than global FAK inhibitors, and ZINC4085554 may be an important step towards the inhibition of metastasis.

\section{Introduction}

Metastasis is the primary cause of mortality in patients with cancer; however, it is poorly understood due to its complexity and multiple step nature (1). Adhesive interactions between the circulating tumor cells and the target organ are an important early step in the formation of metastasis at secondary sites $(1,2)$. Since adhesive interactions are the first step of cancer metastasis, it is imperative to understand the mechanisms contributing to this process, as inhibiting this early metastatic step will prevent subsequent metastatic processes, including migration, intravasation, invasion and new metastatic growths (3-5).

Disseminated cancer cells exhibit varying degrees of adhesive potential, which is dependent on environmental factors, including serum growth factors, extracellular matrix composition, physical forces, temperature and humidity $(6,7)$. In particular, mechanical forces generated in the circulation during laparoscopic insufflation, surgical manipulation, postoperative abdominal third spacing and passage through the lymphatics and circulation may increase the adhesive potential of a wide variety of cancer cells from colonic cancer (8), breast cancer (9), head and neck squamous cell cancer (10) and sarcoma types (11). The stimulation of cancer cell adhesion by extracellular pressure therefore represents an interesting mechanism that regulates cancer cell adhesiveness and a probe to delineate the relevant intracellular signal pathway, in the hope that the identified novel targets can be blocked to inhibit metastasis. Our previous investigation reported that this effect is statistically significant at $10 \mathrm{mmHg}$, and was maximal at $15 \mathrm{mmHg}$ (12), yet increased pressures do not yield an increased effect, at least in vitro (12). Since the effect is maximal at $15 \mathrm{mmHg}$ and as $15 \mathrm{mmHg}$ is the pressure most commonly used to inflate the abdomen in laparoscopic surgery (13), $15 \mathrm{mmHg}$ was selected to investigate increased pressures above ambient in the present study.

Mechanical forces, such as increased extracellular pressure and shear stress, activate cytoskeletally-dependent and cytoskeletally-independent pathways that converge on the activation of focal adhesion kinase (FAK) (14). Although FAK-Tyr-397 phosphorylation is generally conceived as the 
initial step in FAK activation (15), it was observed that FAK activation in cancer cells in response to physical forces requires the upstream activation of Akt1 at the catalytic region Ser-473, yet not at the regulatory site Thr-308, which then binds to FAK and induces its phosphorylation at serine 517,601 and 695 and threonine 600 (16,17). As FAK activation does not generally require $\mathrm{Akt}$, this novel interaction may be a promising target for pharmacologic blockade, which may not interfere with other aspects of FAK signaling, and therefore provides a more specific targeting mechanism than the global FAK inhibitors currently in trials (18-21). Serial truncation of the FAK FERM domain demonstrated that FAK-Aktl binding occurs in sub domain 1-126 amino acids (22). Further serial truncation of this domain narrowed down to 33 amino acids NT1-2-2 FERM (amino acid sequence 94-126 of FERM) subdomain of FAK is required for Akt1 binding (23), and further studies condensed this further to the 7 amino acid sequence 113LAHPPEEQ119, which is a short helical structure (24). Adenoviral expression of this peptide in colon cancer cells prevents activation of FAK in response to extracellular pressure, attenuates FAK-Akt1 interaction, and inhibits the pressure-induced adhesion of colon cancer cells to a collagen I substrate (24). Furthermore, cells transiently overexpressing this peptide are less adherent in murine surgical wound tumor recurrence models, decreasing tumor incidence and improving subsequent survival (24).

However, small molecules may be more advantageous as therapeutic agents than peptides with respect to half-life, cell permeability and stability $(25,26)$. For instance, small molecules are more stable than peptides in various physiological environments, including those at varying $\mathrm{pH}$, or containing degradation enzymes, or with different cation or anion concentrations $(25,26)$. In a previous preliminary study, a three dimensional ligand-based virtual screen was performed to identify small molecules that may mimic the structure of this peptide within FAK, and reported that two such molecules, ZINC04085549 and ZINC4085554, could inhibit the pressure-induced adhesion of SW620 colon cancer cells to collagen I (27). The aim of the present study was to examine the effects of ZINC4085554 on adhesion to collagen I in vitro and to wound extracellular matrix (of which type I collagen is the dominant collagen) in vivo. The promising molecule, ZINC4085554 was selected, to delineate the mechanism of this anti-adhesive effect, and examine the hypothesis that ZINC4085554 reduces cancer cell adhesion by inhibiting the interaction of Akt1 with FAK, as well as the additional hypothesis that this molecule would not interfere with more conventional FAK signaling. The results of the present study confirmed that ZINC4085554 can inhibit cancer cell adhesion not only to purified proteins in cell culture, but also to intact tissues in living mice.

\section{Materials and methods}

Cell culture and Reagents. The SW620 human colon cancer cell line was purchased from the American Tissue Culture Collection (Manassas, VA, USA), and were routinely cultured in Leibovitz's L-15 (L15) media (cat. no. 11415-064; Thermo Fisher Scientific, Inc., Waltham, MA, USA) containing penicillin and streptomycin (cat. no. 15140-122; Thermo Fisher Scientific, Inc.) at $100 \mathrm{U} / \mathrm{mL}$ and $100 \mu \mathrm{g} / \mathrm{ml}$, respectively, and $10 \%$ fetal bovine serum (FBS). Cells placed at $37^{\circ} \mathrm{C}$ in a humidified incubator containing $5 \% \mathrm{CO}_{2}$.

Extracellular pressure treatment. Cells (50,000 cells/well) were seeded into 24 well plates that were then subjected to $15 \mathrm{mmHg}$ increased extracellular pressure for $30 \mathrm{~min}$ at $37^{\circ} \mathrm{C}$ in a mixture of $5 \% \mathrm{CO}_{2}$ with $95 \%$ room air in a pre-warmed air tight box with inlet and outlet valves and a pressure gauge, as described previously $(17,24,27)$. It has been previously reported that this technique, if the box is appropriately pre-warmed, allows for the control temperature, pressure and oxygen partial pressure within the cell culture medium. A preliminary study demonstrated the ability to maintain constant temperature and pressure conditions of $2^{\circ} \mathrm{C}$ and $1.5 \mathrm{mmHg}$, respectively, using this method (12).

Adhesion assay. Our previous studies performed cell counting via optical microscope $(23,28)$ and Tag-it dye (Tag-it Violet Proliferation Cell Tracking Dye; Biolegend, Inc., San Diego, CA, USA) staining method $(24,27)$ for cell counting and have revealed that dye staining methods are more objective as they do not require the observer to select which microscopic fields to count and are more accurate as they compensate for any unevenness of distribution of cells across the well. Therefore, the adherent cells were not imaged, and only their optical densities were measured. Our studies $(24,27)$ and other studies $(29,30)$ routinely used such techniques to measure adhesion.

The adhesion assay was performed in petri dishes pre-coated with type I collagen (Sigma-Aldrich; Merck KGaA, Darmstadt, Germany), as previously described (12). Cells treated at $37^{\circ} \mathrm{C}$ for $1 \mathrm{~h}$ with vehicle [0.1\% dimethyl sulfoxide (DMSO)] or ZINC4085554 (10, 25 and $50 \mu \mathrm{M})$ were seeded into collagen I pre-coated 24 well plate at 50,000 cells/well. One plate was maintained at ambient pressure while the other was subjected to $15 \mathrm{mmHg}$ pressure, each in $5 \% \mathrm{CO}_{2}$ at $37^{\circ} \mathrm{C}$. At $30 \mathrm{~min}$, the non-adherent cells were washed away PBS. The remaining adherent cells were stained with MTS (CellTiter 96 Aqueous One Solution Cell Proliferation Assay; Promega Corporation, Madison, WI, USA) at $37^{\circ} \mathrm{C}$ for $1 \mathrm{~h}$ and absorbance was measured at $490 \mathrm{~nm}$.

Western blotting and co-immunoprecipitation (co-IP). As the signal cascade examined herein occurs in suspended cells prior to adhesion, the majority of signaling studies were performed in dishes pre-coated with $1 \%$ heat inactivated bovine serum albumin (BSA) (Sigma-Aldrich; Merck KGaA) to prevent adhesion. SW620 cells were treated with vehicle (0.1\% DMSO) or ZINC4085554 $(50 \mu \mathrm{M})$ and subjected to ambient and $15 \mathrm{mmHg}$ pressure in $1 \%$ heat inactivated BSA pre-coated dishes, for $30 \mathrm{~min}$ at $37^{\circ} \mathrm{C}$ in $5 \% \mathrm{CO}_{2}$. The cells were then collected and lysed in lysis buffer $(50 \mathrm{mM}$ Tris, $150 \mathrm{mM} \mathrm{NaCl}, 1 \mathrm{mM}$ EDTA, $1 \mathrm{mM}$ EGTA, 1\% Triton-X-100, $1 \%$ deoxycholic acid, $0.1 \%$ SDS, $10 \%$ glycerol, and protease and phosphatase inhibitors) and concentration of protein was estimated by bicinchoninic acid. Proteins $(50 \mu \mathrm{g})$ were separated by $10 \%$ SDS-PAGE and transferred onto nitrocellulose membranes. Membranes were blocked with blocking buffer (Odyssey Blocking Buffer; LI-COR Biosciences, Lincoln, $\mathrm{NE}$, USA) at room temperature for $1 \mathrm{~h}$ and immunoblotted 
at $4{ }^{\circ} \mathrm{C}$ overnight with primary antibodies as follows: FAK (1:1,000; cat. no. 05-537, clone 4.47; mouse monoclonal; Merck KGaA, Darmstadt, Germany), FAK-Try-397 (1:1,000; cat. no. ab81298; rabbit monoclonal; Abcam, Cambridge, UK), Akt1 (1:1,000; cat. no. 2967, clone 2H10; mouse monoclonal; Cell Signaling Technology, Inc., Danvers, MA, USA), Akt1-Ser-473 (1:1,000; cat. no. 9276; mouse monoclonal; Cell Signaling Technology, Inc.) and Akt-Thr-308 (1:1,000; cat. no. 9275; Cell Signaling Technology, Inc.). Infrared fluorescence dye (IRDye) detection method was used to visualize the immunoblots. IRDye conjugated secondary antibodies were the rabbit IRDye 680 (cat. no. P/N 925-68073), rabbit IRDye 800 (cat. no. P/N 925-32213), mouse IRDye 680 (cat. no. $\mathrm{P} / \mathrm{N}$ 925-68072) and mouse IRDye 800 (cat. no. P/N 925-32212). All secondary antibodies were purchased from LI-COR Biosciences, used at 1:15,000 and incubated at room temperature for $1 \mathrm{~h}$. The IRDye 680 and IRDye 800 conjugated secondary antibodies were used to detect the signal at $700 \mathrm{~nm}$ and $800 \mathrm{~nm}$ channel, respectively, using an Odyssey Fc Imaging system (LI-COR Biosciences). Images were captured and analyzed using LI-COR software Image Studio Lite v.5.x (LI-COR Biosciences).

For comparative studies of the effects of ZINC4085554 on collagen I stimulated FAK-Tyr-397 phosphorylation in SW620 cells, the dishes were coated with $1 \%$ heat inactivated BSA or collagen I.

For the co-IP studies, cells were transfected using Lipofectamine ${ }^{\mathrm{TM}} 2000$ transfection reagent (Thermo Fisher Scientific, Inc.) with $2 \mu \mathrm{g}$ control hemagglutinin (HA), Tag-plasmid DNA (pcDNA3; Addgene, Inc., Cambridge, MA, USA) and HA-FAK plasmid DNA construct (HA-FAKpcDNA3; kindly donated by Dr. Guan; Department of Cancer Biology, University of Cincinnati College of Medicine, Cincinnati, OH, USA) to overexpress FAK (PTK2) tagged with HA. After $72 \mathrm{~h}$ transfection, these cells were treated with vehicle (0.1\% DMSO) and $50 \mu \mathrm{M}$ ZINC4085554 in suspension and subjected to ambient and $15 \mathrm{mmHg}$ pressure for $30 \mathrm{~min}$ in $1 \%$ heat inactivated BSA coated dishes at $37^{\circ} \mathrm{C}$ in $5 \% \mathrm{CO}_{2}$. These cells were then lysed in non-denaturing lysis buffer (as aforementioned without SDS). A total of 1-2 mg total protein was used for the immunoprecipitation. The pre-cleared total protein was incubated with anti-HA antibody (1:200; cat. no. 05-904; mouse monoclonal; Merck $\mathrm{KGaA})$ at $4^{\circ} \mathrm{C}$ overnight. Normal mouse IgG (1:200; cat. no. SC-2025; Santa Cruz Biotechnology, Inc., Dallas, TX, USA) was used as a control antibody for the co-IP. Protein G plus/protein A agarose suspension beads (Merck KGaA) were added to the lysate containing the anti-HA antibody (1:200; cat. no. 05-904, mouse monoclonal; Merck KGaA) and incubated for additional $2 \mathrm{~h}$ at $4^{\circ} \mathrm{C}$. The beads were washed three times with the same lysis buffer and the protein was eluted in 6X Laemmli buffer (Morganville Scientific, Morganville, NJ, USA). The eluted protein samples from the original 1-2 mg of protein were resolved by $10 \%$ SDS-PAGE, transferred onto nitrocellulose membranes, and immunoblotted with anti-HA antibody (1:1,000; cat. no. 3724 (C29F4); rabbit; Cell Signaling Technology, Inc.) and/or anti-Akt1 antibody (1:1,000; cat. no. 2967 (2H10); mouse; Cell Signaling Technology, Inc.). The total lysates loaded on gel and western blotted for HA-FAK and Aktl served as input control for the co-IP. Densitometric quantitation was performed with Kodak Scientific Imaging Systems 1D v.3.5.4 or LI-COR imaging software (LI-COR Biosciences).

Surgical wound adhesion assay. This assay was designed to study cancer cell adhesion, which is the first step in cancer recurrence at surgical wounds (2). We previously extensively validated this assay $(4,24,31)$. A total of 24 male mice (8-10 week old) were included in this study. Mice were housed in temperature-controlled rooms with $12 \mathrm{~h}$ light and $12 \mathrm{~h}$ dark cycle at $22-24^{\circ} \mathrm{C}$ and $50-60 \%$ humidity. All animals had free access food and water. As the animals were sacrificed post-adhesion and the non-adherent cells were washed away, wound healing was not studied in these animals.

The present study was approved by the University of North Dakota institutional animal use committee, and performed using a technique previously described (4,24). SW620 cells were treated with DMSO or $50 \mu \mathrm{M}$ ZINC4085554 at $37^{\circ} \mathrm{C}$ for $1 \mathrm{~h}$ in an incubator. Following incubation, cells ( $\sim 2$ million/well of a 24 -well plate) were trypsinized with $1 \mathrm{ml}$ of $0.05 \%$ trypsin, neutralized with $1 \mathrm{ml}$ of culture medium containing $10 \% \mathrm{FBS}$, and labeled with $50 \mu \mathrm{M}$ Tag-it dye in PBS at $37^{\circ} \mathrm{C}$ for $20 \mathrm{~min}$ in suspension (Tag-it Violet Proliferation Cell Tracking Dye; Biolegend, Inc.). PBS containing 5\% FBS was added to quench the excess dye and then cells were pelleted and suspended in complete L15 media. The suspended cells were treated with DMSO or $50 \mu \mathrm{M}$ ZINC4085554 and maintained at ambient or $15 \mathrm{mmHg}$ increased extracellular pressure as aforementioned. Following pressure treatment, the cells were collected and counted. C57BL/6 mice were anesthetized intraperitoneally using ketamine $(100 \mathrm{mg} / \mathrm{kg})$, xylazine $(10 \mathrm{mg} / \mathrm{kg})$ and aceproject maleate $(3 \mathrm{mg} / \mathrm{kg})$ cocktail. A small incision was made at the thigh and armpit region. The 400,000 cells in $30 \mu \mathrm{l}$ PBS were seeded for each group in these surgical wounds and permitted to adhere for $30 \mathrm{~min}$. The non-adherent cells were washed away with $50 \mu$ l PBS five times. The mice were then euthanized by cervical dislocation and the wounds were completely excised along with a margin of unwounded tissue. The excised tissue was digested using type I collagenase (Thermo Fisher Scientific, Inc.) and a single cell suspension was prepared. These cells were fixed using a FoxP3/Transcription factor fixation/permeabilization kit from eBioscience (Thermo Fisher Scientific, Inc.) at $4^{\circ} \mathrm{C}$ for $30 \mathrm{~min}$ and acquired using a LSRII flow cytometer (BD Biosciences, San Jose, CA, USA), gating so as to count only the Tag-it-labeled cells that had been adherent to the surgical wounds at time of sacrifice. No fluorophore kit or antibody was required since cells could be detected by the Tag-it label. FlowJo software v.10 (FlowJo LLC, Ashland, OR, USA) was used to analyze the data. It should be noted that eBioscience Foxp3/Transcription factor fixation/permeabilization concentrate and diluent is the name of a fixation/permeabilization buffer (eBioscience; Thermo Fisher Scientific, Inc.). Although originally used to investigate Foxp3, it has a general purpose as a fixation permeabilization buffer and it was determined to be beneficial to permeabilize digested surgical wounds so that flow cytometry can be conducted to quantitate pre-labeled adherent cancer cells within the wound. The use of this buffer for flow cytometry has been discussed previously $(32,33)$. 
Statistical analysis. All assays were performed within the linear range. Data was normalized against the vehicle (0.1\% DMSO)-treated ambient pressure condition, and represented as the mean \pm standard error of the mean. SigmaPlot v.13 software (Systat Software, Inc., San Jose, CA, USA) was used for statistical analysis. Unpaired student's t-test was used to analyze the data. $\mathrm{P}<0.05$ was considered to indicate a statistically significant difference.

\section{Results}

ZINC4085554 inhibits pressure-stimulated adhesion of SW620 cells. The present study first confirmed our previous cell adhesion results with ZINC4085554. The data revealed that $15 \mathrm{mmHg}$ of pressure increases $24.1 \pm 4.9 \%$ of the adhesion vs. ambient pressure in DMSO-treated SW620 cells. ZINC4085554 at $10 \mu \mathrm{M}$ did not affect the adhesion of SW620 cells at ambient pressure or prevent the stimulation of adhesion by $15 \mathrm{mmHg}$ extracellular pressure $(33.9 \pm 8.6 \%$ increase in ambient vs. pressure). In contrast, at $25 \mu \mathrm{M}$, an attenuation of the pressure effect was observed $(12.3 \pm 10.6 \%)$, and at $50 \mu \mathrm{M}$ the pressure effect on adhesion was blocked further $(11.2 \pm 7.9 \%)$, reducing adhesion compared with that observed at ambient pressure (Fig. 1). Therefore, although an effect was observed at $25 \mu \mathrm{M}, 50 \mu \mathrm{M}$ was selected for the remainder of this investigation due to its increased potency.

Pressure stimulates FAK-Tyr-397 phosphorylation yet not Aktl-Ser-473, and is inhibited by ZINC4085554, while Akt-Thr-308 remains unaffected by pressure and/or ZINC4085554. It has previously been reported that Akt1 phosphorylation at Ser-473 (but not at Thr-308) precedes and is required for FAK-Tyr-397 phosphorylation in the stimulation of cancer cell adhesion by pressure $(16,28)$, although FAK-Tyr-397 phosphorylation usually does not require Akt1 in other settings (34). The present study therefore examined the effect of $50 \mu \mathrm{M}$ ZINC4085554 on FAK and Akt1 phosphorylation. There was a FAK-Tyr-397 increase of $18.7 \pm 3.2 \%$ in pressure-stimulated SW620 cells (Fig. 2A and B) and a $71.2 \pm 16.9 \%$ increase in Akt1-Ser-473 phosphorylation (Fig. 2C and D) in comparison to ambient pressure SW620 cells treated with the DMSO vehicle. Consistent with our previous findings, the Akt-Thr-308 remains unaltered by pressure (16) or ZINC4085554 (Fig. 2E and F). ZINC4085554 did not affect basal FAK-Tyr-397 or Akt1 phosphorylation at ambient pressure. However, ZINC4085554 did inhibit the stimulation of FAK-Tyr-397 phosphorylation at $15 \mathrm{mmHg}$, yet did not affect pressure-stimulated Akt1 phosphorylation at Ser-473 (Fig. 2), indicating that ZINC4085554 inhibits FAK-Tyr-397 phosphorylation downstream of Akt1.

ZINC4085554 inhibits the pressure-stimulated interaction of FAK and Akt1. As ZINC4085554 may block pressure-induced FAK-Tyr-397 phosphorylation without affecting upstream Akt1 activation at Ser-473, the present study then examined the effects of ZINC4085554 on interactions of FAK-Akt1 following exposure to increased extracellular pressure. Co-IP studies demonstrated a $29.5 \pm 13.2 \%$ increase in the pull-down fractions of Akt1 using HA-FAK vs. ambient DMSO in cells treated with DMSO at $15 \mathrm{mmHg}$ increased pressure. In

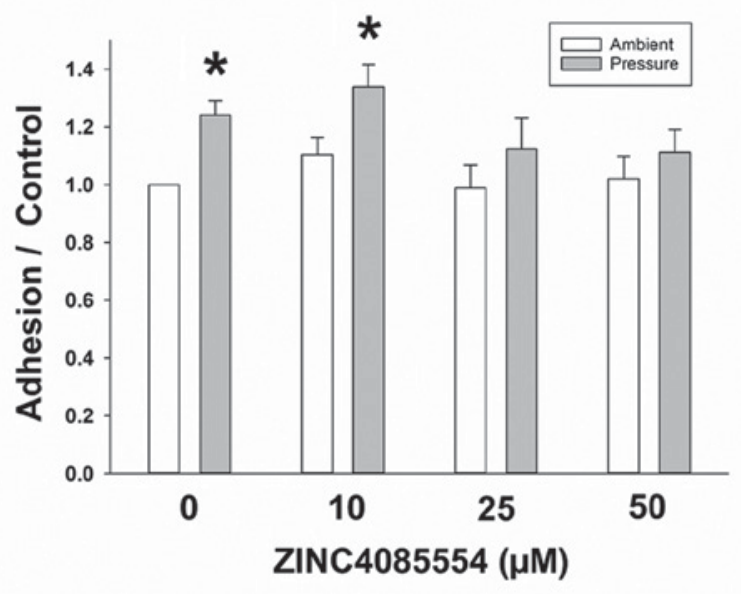

Figure 1. ZINC4085554 inhibits pressure-stimulated adhesion of SW620 cells SW620 cells were treated with vehicle ( $0.1 \%$ dimethyl sulfoxide), and 10, 25 and $50 \mu \mathrm{M}$ ZINC4085554 and allowed to adhere to collagen I for $30 \mathrm{~min}$ at ambient or $15 \mathrm{mmHg}$ increased pressure. $(\mathrm{n}=5-9)$. ${ }^{*} \mathrm{P}<0.05$ vs. ambient.

contrast, in SW620 cells treated with $50 \mu \mathrm{M}$ ZINC4085554 and exposed to $15 \mathrm{mmHg}$ increased pressure did not stimulate the increased pull-down of Akt1 using HA-FAK; however, yielded Akt1 pull-down similar to that observed at ambient pressure (Fig. 3). This indicates that ZINC4085554 interferes with the pressure-stimulated interaction of FAK-Akt1.

Collagen I stimulated FAK-Tyr-397 phosphorylation is unaffected by treatment with ZINC4085554. Although the present study focused on the stimulation of FAK-Tyr-397 phosphorylation by increased extracellular pressure in suspended cancer cells prior to adhesion to a matrix substrate, FAK phosphorylation is much more commonly studied in epithelial cells in response to adhesion (15,35-39). This canonical pathway involves integrin heterodimer engagement with extracellular matrix proteins, and shifts of integrin conformation across the cell membrane that result in FAK phosphorylation within the focal adhesion complex (36). This pathway does not require Akt for FAK phosphorylation (34), and the reason the present study focused on inhibiting Akt-FAK interaction is that an agent that specifically blocks pressure-induced Akt-FAK interaction should prevent pressure-induced downstream FAK activation in cancer cells without interfering with the more common FAK activation in other cells and tissues that is engendered by cell-matrix interactions. Therefore, the present study sought to address the specificity of the effect of ZINC4085554 on pressure-stimulated FAK phosphorylation.

The effects of ZINC4085554 on pressure-stimulated FAK-Tyr-397 phosphorylation were compared with the induction of FAK-Tyr-397 phosphorylation by adhesion to type I collagen by examining the effect of ZINC4085554 on collagen I stimulated FAK-Tyr-397 phosphorylation.

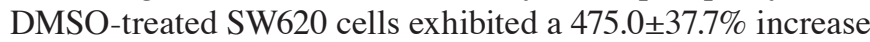
in FAK-Tyr-397 phosphorylation on collagen I vs. BSA coated plates. The same effect was observed $(550.8 \pm 99.7 \%$ increase in FAK-Tyr-397 phosphorylation on collagen I vs. BSA coated plates) even in the presence of $50 \mu \mathrm{M}$ ZINC4085554 (Fig. 4). Consequently, collagen I induced FAK-Tyr-397 phosphorylation was unaffected by ZINC4085554, in contrast to the 

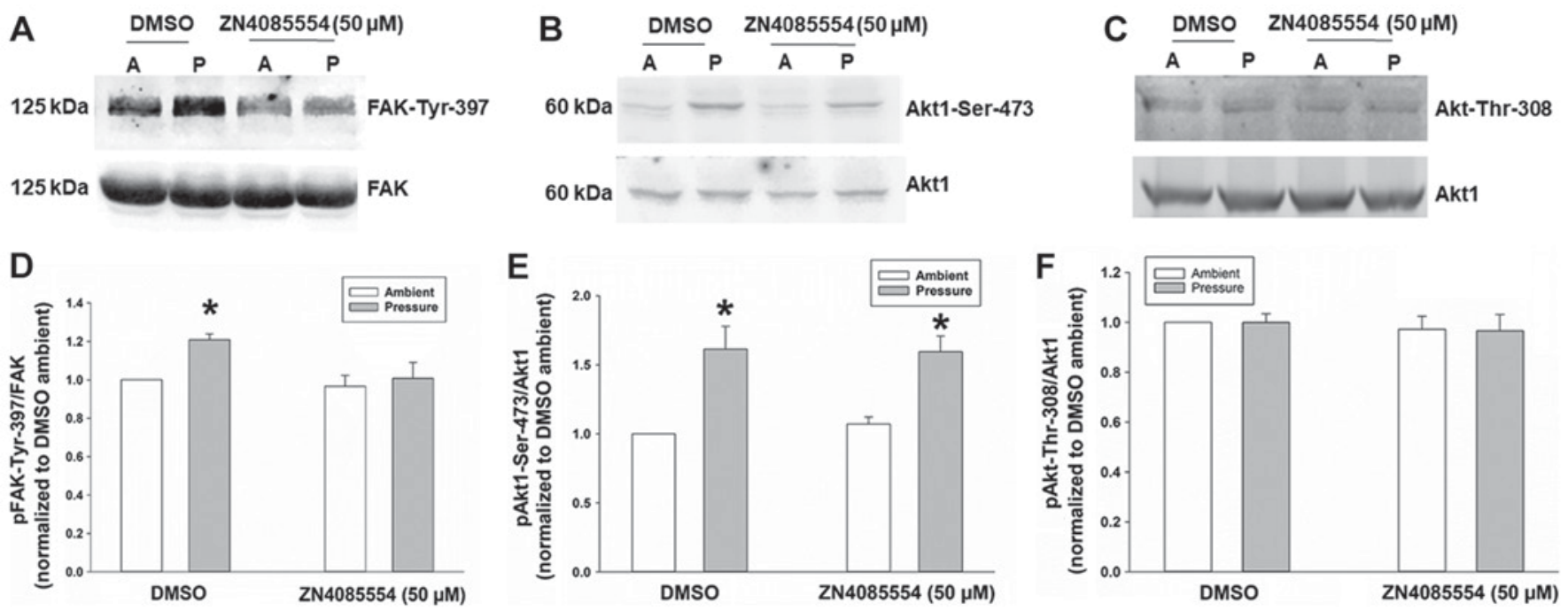

Figure 2. ZINC4085554 inhibits pressure-stimulated FAK-Tyr-397 yet not Akt1-Ser-473 phosphorylation, and Akt-Thr-308 remains unaffected by pressure and/or ZINC4085554. SW620 cells were treated with vehicle (0.1\% DMSO) or $50 \mu \mathrm{M}$ ZINC4085554 and subjected to ambient or $15 \mathrm{mmHg}$ increased extracellular pressure for $30 \mathrm{~min}$. (A) Representative blots probed for FAK-Tyr-397 and total FAK. (B) Densitometric quantitation of the blots in A. (C) Representative blots probed for Akt1-Ser-473 and total Akt1. (D) Densitometric quantitation of the blots in C. (E) Representative blots probed for Akt-Thr-308 and total Akt1. (F) Densitometric quantitation of the blots in $\mathrm{E}$. ( $\mathrm{n}=5-8)$ " $\mathrm{P}<0.05$ vs. ambient. A, ambient; P, pressure; FAK, focal adhesion kinase; DMSO, dimethyl sulfoxide.

A

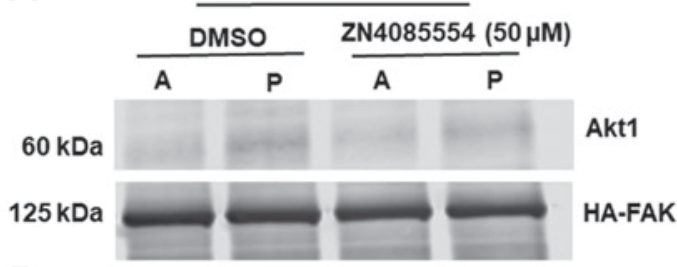

B

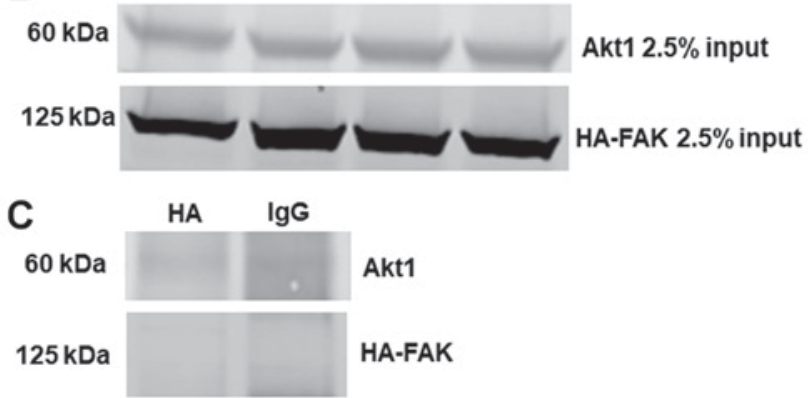

D

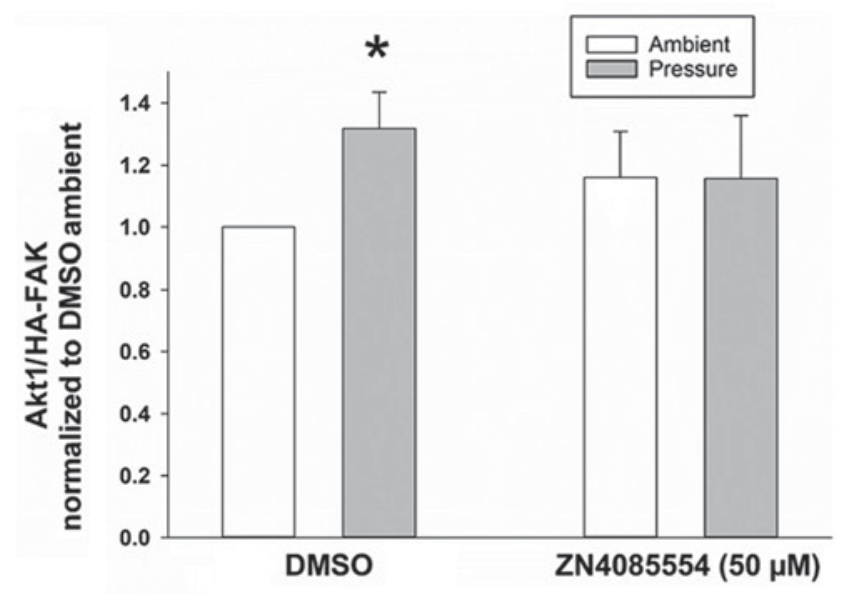

Figure 3. ZINC4085554 obstructs FAK-Akt1 interaction. SW620 cells were transfected with empty vector HA plasmid DNA or HA-FAK plasmid DNA. These cells were treated with vehicle (0.1\% DMSO) or $50 \mu \mathrm{M}$ ZINC4085554 and exposed to ambient or $15 \mathrm{mmHg}$ increased pressure. The lysates were prepared and co-IP was performed using anti-HA antibody. Cells transfected with HA plasmid DNA served as a transfection control (HA lane) while normal mouse IgG was used as a co-IP control (IgG lane). (A) Representative blots probed for HA-FAK and total Aktl for co-IP, (B) total lysate as an input and (C) o-IP controls HA and IgG. (D) Densitometric quantitation of the co-IP blots ( $\mathrm{n}=10)$ " $\mathrm{P}<0.05$ vs. ambient. A, ambient; $\mathrm{P}$, pressure; FAK, focal adhesion kinase; DMSO, dimethyl sulfoxide; co-IP, co-immunoprecipitation.

induction of FAK-Tyr-397 phosphorylation by increased extracellular pressure.

ZINC4085554 inhibits the pressure-stimulated surgical wound adhesion in mice models. The present study then sought to confirm the effect of ZINC4085554 on the adhesion of SW620 cells to more biologically complex intact tissues in the surgical wounds of mice. SW620 cells treated with DMSO and/or ZINC4085554 at ambient or $15 \mathrm{mmHg}$ increased pressure were allowed to adhere to surgical wounds in mice, prior to non-adherent cells were washed away, in order to mimic the cancer cell adhesion that occurs in surgical fields during cancer resection, in which shed cancer cells initially may adhere locally prior to subsequent irrigation of the surgical field. An $84.4 \pm 42.1 \%$ increase was observed in the wound adhesion of the DMSO-treated SW620 cells exposed to $15 \mathrm{mmHg}$ pressure vs. cells maintained at ambient pressure. In contrast, the cells treated with $50 \mu \mathrm{M}$ ZINC4085554 did not exhibit significantly increased adhesion following stimulation by $15 \mathrm{mmHg}$ increased pressure yet rather displayed adhesion to surgical wounds similar to that observed by cells exposed to ambient pressure (Fig. 5). 
A

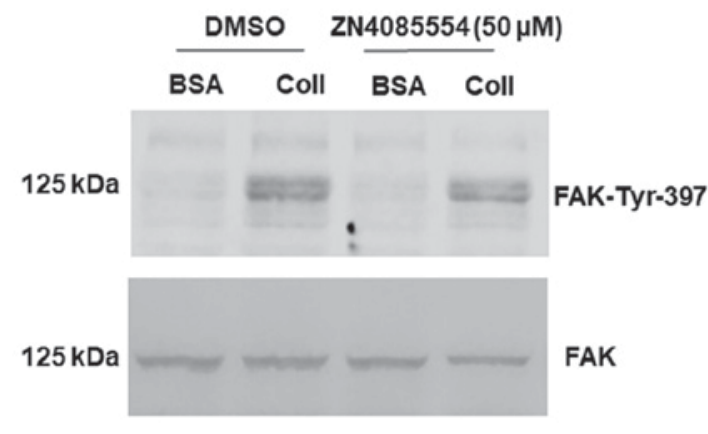

B

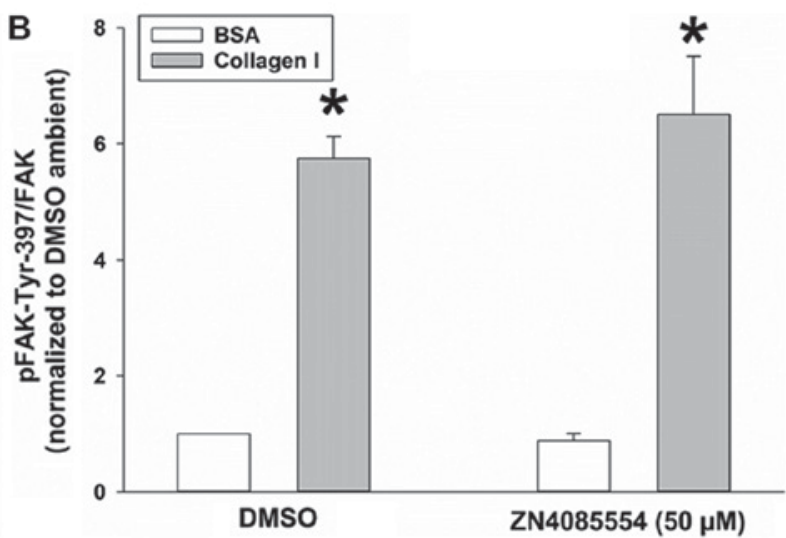

Figure 4. ZINC4085554 does not block FAK-Tyr-397 phosphorylation stimulated by adhesion to type I collagen. SW620 cells were treated with vehicle (0.1\% DMSO) or $50 \mu \mathrm{M}$ ZINC4085554 and seeded into dishes pre-coated with either $1 \%$ heat inactivated BSA or collagen I for $1 \mathrm{~h}$. (A) Representative blots probed for FAK-Tyr-397 and total FAK. (B) Densitometric quantitation of the blots ( $\mathrm{n}=5$ ). ${ }^{*} \mathrm{P}<0.05$ vs. BSA. FAK, focal adhesion kinase; DMSO, dimethyl sulfoxide; BSA, bovine serum albumin; p, phospho.

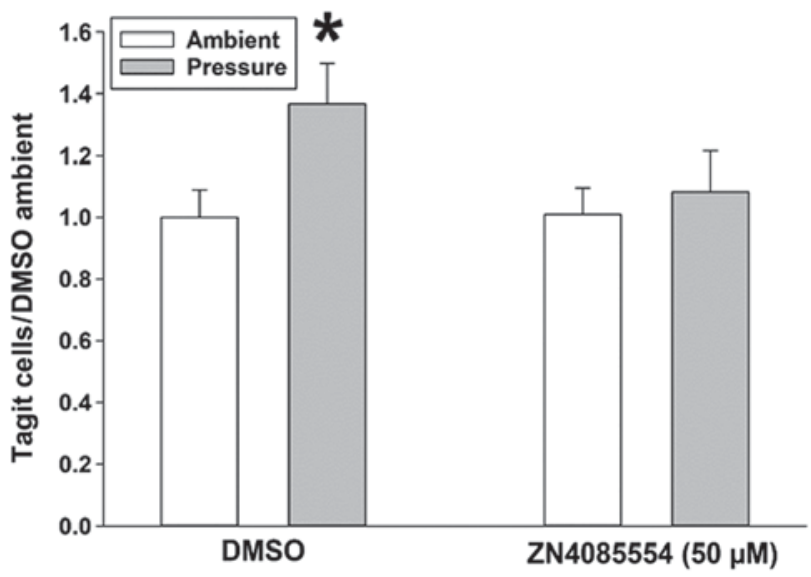

Figure 5. ZINC4085554 inhibits the adhesion of SW620 cells to surgical wounds in mice. Tag-It-labelled SW620 cells were treated with vehicle (0.1\% DMSO) or $50 \mu \mathrm{M}$ ZINC4085554 and cultured for $30 \mathrm{~min}$ at ambient or $15 \mathrm{mmHg}$ increased pressure. These cells were then allowed to adhere to the surgical wounds for $30 \mathrm{~min}$. Non-adherent cells were washed away, wound tissue was excised, and the number of Tag-It-labeled cells were assessed from the wound using flow cytometry. Bar diagram represents the normalized data for adherent cells in each condition ( $\mathrm{n}=24,{ }^{*} \mathrm{P}<0.05$ vs. ambient). DMSO, dimethyl sulfoxide.

\section{Discussion}

Mechanical forces stimulate the cancer cell adhesiveness required for metastasis $(8,40)$. FAK-Akt1 interaction serves a crucial role in $15 \mathrm{mmHg}$ pressure above ambient stimulated adhesion of colon cancer cells (24). The interaction between Akt1 and FAK represents an opportune target for intervention, which unlike conventional FAK or Akt inhibitors, may specifically ablate the mechanosensitive signaling pathways that promotes cancer cell adhesion without interfering with other FAK or Akt signaling in host tissues. Building upon a preliminary in silico screening and confirmation that at least two of the candidate molecules may inhibit cancer cell adhesion (27), the present study demonstrated that ZINC4085554 inhibits pressure-stimulated FAK activation; however, it does not prevent the upstream activation of Akt1 by increased extracellular pressure or the activation of FAK by more conventional outside-in signaling induced by adhesion to type I collagen, a dominant interstitial matrix component (41). Instead, ZINC4085554 attenuated FAK-Akt1 interaction. Lastly, this molecule inhibited the $15 \mathrm{mmHg}$ pressure-stimulated adhesion of colon cancer cells in the surgical wounds of mice.

FAK activation is one of the central steps in the adhesive processes of cancer cells, and also critical to numerous other signaling pathways, including cell migration, response to cyclic strain and response to growth factors $(15,18,42)$. A wide variety of scaffolding proteins, adapter molecules, signaling messengers and transmembrane proteins regulate FAK while FAK also regulates its own activation and deactivation via dimerization and auto-phosphorylation $(35,43,44)$. While FAK activation in response to extracellular forces requires Akt $(17,37,45)$, FAK activation in other contexts occurs independently of Akt (34), and Akt may or may not be activated downstream of FAK, depending on the context $(34,38,46)$. The phosphorylation of Akt1 at Thr-308 and Ser-473 has previously been reported to potentiate its full activation. PDK1 phosphorylates Akt1 at Thr-308 in the catalytic region or activation loop and this phosphorylation does increase Akt1 activity (47). However, mTORC2 phosphorylation of Akt1 at Ser-473, in the C-terminal hydrophobic motif, is hypothesized to be required for Akt1 maximal activation (47). The literature indicates that certain signaling pathways/proteins exclusively phosphorylate each site for Akt activation. For instance, tumor necrosis factor- $\alpha$ exclusively phosphorylates Akt at Akt-Ser-473, but not at Thr-308 (48). Similarly, glutamate-exposed neurons exhibit Akt phosphorylation at Akt-Ser-473, yet not at Thr-308 (49). We have previously reported that exposure to increased extracellular pressure also results in the phosphorylation of Akt-Ser-473, but not of Akt-Thr-308 (16). The results of the present study are consistent with the previous observation of the absence of Akt-Thr-308 phosphorylation in response to extracellular pressure.

The observation that ZINC4085554 does not prevent FAK activation in response to adhesion is not unexpected. FAK is activated in response to a diverse range of stimuli, including cell-matrix interactions $(36,39)$ and transactivation by the epidermal growth factor receptor $(42,50)$. These canonical pathways do not require Akt to interact with $\operatorname{FAK}(21,34,51,52)$. 
For instance, adhesion itself engages integrin heterodimers with moieties on the extracellular matrix, inducing a conformational shift in the $\beta-1$ integrin subunit that transmits across the cell membrane and is eventually associated with FAK-Tyr-397 phosphorylation without involving $\mathrm{Akt}$, and this pathway has been extensively reviewed $(21,34,36,51)$. In contrast, the activation of FAK in cancer cells in response to extracellular pressure requires a preliminary FAK-Akt interaction, which makes FAK-Akt interaction an attractive target to specifically block pressure-induced FAK activation in cancer cells without interfering with other FAK signaling that may be notable in other tissues and organs within the patient (17,22-24,27). The results of the present study are consistent with this model, since ZINC4085554 prevents FAK-Tyr-397 phosphorylation in suspended cells in response to pressure but does not prevent the FAK-Tyr-397 phosphorylation that occurs post- and in response to adhesion to a matrix.

Indeed, these results further indicate that ZINC4085554 is neither a conventional FAK inhibitor as it permits FAK activation in response to adhesion, nor a conventional Akt inhibitor, as it allows Akt activation at Ser-473 in response to increased extracellular pressure. ZINC4085554 may interfere with the binding of Aktl to FAK after Aktl has been activated in the setting of increased extracellular pressure, and thereby more specifically prevents FAK activation by increased extracellular pressure while allowing FAK activation by a different pathway. This indicates the possibility that molecules based on ZINC4085554 may exhibit less off-target specificity than conventional FAK or Akt inhibitors by allowing other FAK and Akt signaling to continue unabated. It is notable that physical force effects on other cell types may invoke different signaling pathways. For instance, increased extracellular pressure consistent with the edema in inflamed tissues stimulates macrophage phagocytosis by a pathway that involves the activation of Akt 2 rather than Akt1, and the inhibition of FAK rather than its stimulation (53).

The concept that ZINC4085554 inhibits adhesion by interfering with FAK-Akt1 interaction would be consistent with our original hypothesis that ZINC4085554 resembles the subdomain of FAK to which Akt1 binds. ZINC4085554 was identified by in silico screening as a small molecule resembling a two site mutant peptide derived from a helical subdomain in FAK that was revealed to compete with FAK for binding to Aktl (27). As FAK itself dimerizes, it will be notable to determine in future studies whether ZINC4085554 binds to FAK itself, thereby occupying the Akt1 binding site, or whether ZINC4085554 binds to Akt1, occupying the FAK binding site. This awaits further study.

The present study further confirmed that ZINC4085554 not only inhibits cancer cell adhesion to a type I collagen substrate in vitro but also to the biological tissues in a murine surgical wound. This was notable as metastatic adhesion must occur to a complex tissue in vivo offering multiple binding sites beyond those that are present in type I collagen $(1,4)$. It has previously been reported that increased extracellular pressure also stimulates cancer cell adhesion to endothelial cells (54). Although the increase in adherent cell numbers in vitro and to the mouse wounds with extracellular pressure and its blockade by ZINC4085554 may seem small in magnitude, we previously reported that such small changes in cancer cell adhesion translate to substantial changes in subsequent tumor development and survival in mouse models $(4,24,31,55)$. This may be because tumor development is a stochastic process that requires tumor cells to not only adhere, but to subsequently proliferate and elude host immune surveillance (1,3). Others examined changes of similar magnitude in FAK activation and adhesion in other contexts (56-59). For instance, Hic-5 $5^{-/}$;PyMT tumor cells present reduced FAK-Tyr-397 phosphorylation compared to Hic- $5^{+++}$;PyMT cells (56).

ZINC4085554 may not be a therapeutic agent in itself, as the concentration required for efficacy is relatively high and its dinitrobenzene group may raise chemical safety issues (27). However, these studies represent proof of principle that a small molecule can be developed to inhibit cancer cell adhesiveness by interfering with FAK-Akt1 interaction. It may be easier to modify this molecule or similar molecules for therapeutic testing than to develop an approach based upon the previous demonstration that a seven amino acid FAK-derived peptide can reduce adhesiveness (24) since the latter would necessitate solving problems of delivery and stability.

Postoperative tumor recurrence can reflect the emergence of previously unrecognized and therefore unresected tumors that had previously metastasized, yet not grown sufficiently for detection or the dissemination of new tumor cells at the time of surgery that implants and develop into recurrent disease. Such tumor implantation can occur in surgical wounds, or other aspects of the surgical site such as the peritoneum, or distant organs via lymphatic or circulatory dissemination (4,8,60-67). Although there may be differences in the pathophysiology and mechanics underlying local tumor dissemination to wounds to the peritoneum and dissemination through the circulation, it has previously been demonstrated that increased extracellular pressure promotes wound implantation and peritoneal metastasis (4). Shear stress occurs during circulation, and also activates the same pathway (68). Wound implantation is easier to investigate from a methodological standpoint, and was consequently selected for the first attempt to validate the potential effects of ZINC4085554 in vivo. However, further study is warranted to extend these results to the effects of ZINC4085554 on tumor dissemination via the lymphatics or circulation.

It is not always possible to distinguish metastasis detected postoperatively, which was simply not detected preoperatively from postoperative metastasis that reflects surgical dissemination. However, the increased shedding of tumor cells into the portal and peripheral circulation following colon cancer resection is well documented $(67,69)$, and the presence of such tumor cells in the circulation is an adverse prognostic factor $(61,62)$ even if it is not possible determine which recurrences reflect perioperative shedding and which reflect preexisting disease. Additionally, postoperative recurrence of the tumor within the surgically-created wound may represent a consequence of surgery itself. The pivotal New England Journal of Medicine trial comparing laparoscopic and open colectomy for cancer (60) documented a $0.2 \%$ incidence of wound recurrence in the open surgical group and a $0.5 \%$ incidence of wound recurrence following laparoscopic surgery involving increased intraperitoneal pressure, although the study did not have sufficient sample size to demonstrate a statistical difference in wound recurrence between the two groups. Postoperative wound recurrence is a consequence of surgery in the majority 
of cases, as the wound was not present preoperatively, and a previous study described an increased incidence of wound metastasis in increased intraperitoneal pressure surgical procedures modeling laparoscopic surgery in animals (64). This effect disappears when so-called 'gasless laparoscopy' models using traction (negative pressure) to expand the abdomen rather than pressure to inflate it are used (63). While much of this work has been performed on colorectal cancer, it has now become clear that laparoscopic resections for cervical cancer are more prone to recurrence and no longer represent the standard of care $(65,66)$. Therefore, while it may not be possible to distinguish preoperative undetected disease from perioperative tumor spread in any specific patient, it may not to conclude that at least some postoperative metastasis does unfortunately reflect the effects of surgery. Although much work must still be done before human trials could be contemplated for this approach, these studies are aimed at understanding and blocking the pathways by which physical forces engendered either during surgery or during passage through the lymphatics or circulation in the non-surgical patient can activate the adhesion of metastasizing tumor cells, in the hope that this approach may ultimately inhibit tumor metastasis.

In conclusion, the present study demonstrated that a small molecule that mimics a subdomain of FAK can inhibit cancer cell adhesiveness in vitro and in live murine tissue by interfering with Akt1 binding to FAK. As other FAK and Akt1 signaling were not interfered with, such a molecule may well be used therapeutically at higher concentrations than conventional FAK or Akt inhibitors as FAK or Akt signaling would not be expected to be interfered with. Molecules such as ZINC4085554 therefore seem promising targets for further investigation, while these results also further validate our initial in silico structure-based screening strategy by suggesting that ZINC4085554 works by a mechanism consistent with that which was targeted by that strategy.

\section{Acknowledgements}

Not applicable.

\section{Funding}

No funding was received.

\section{Availability of data and materials}

The datasets used and/or analyzed during the present study are available from the corresponding author on reasonable request.

\section{Authors' contributions}

SKM and MDB designed and planned the study. SKM performed all the experiments. EEVD acquired and analyzed the flow cytometry data. SKM and MDB wrote the manuscript and EEVD reviewed it before submission.

\section{Ethics approval and consent to participate}

All animal studies were approved by the University of North Dakota Institutional Animal Use and Care Committee.

\section{Patient consent for publication}

Not applicable.

\section{Competing interests}

The University of North Dakota and Michigan State University have jointly applied for a patent on "inhibiting FAK-AKT interaction to inhibit metastasis" on which MDB is named as a co-inventor. The authors declare that they have no competing interests.

\section{References}

1. Lambert AW, Pattabiraman DR and Weinberg RA: Emerging Biological Principles of Metastasis. Cell 168: 670-691, 2017.

2. Craig DH and Basson MD: Biological impact of mechanical stimuli on tumor metastasis. Cell Cycle 8: 828-831, 2009.

3. Brooks SA, Lomax-Browne HJ, Carter TM, Kinch CE and Hall DM: Molecular interactions in cancer cell metastasis. Acta Histochem 112: 3-25, 2010.

4. Craig DH, Owen CR, Conway WC, Walsh MF, Downey C and Basson MD: Colchicine inhibits pressure-induced tumor cell implantation within surgical wounds and enhances tumor-free survival in mice. J Clin Invest 118: 3170-3180, 2008.

5. Lafrenie RM, Buchanan MR and Orr FW: Adhesion molecules and their role in cancer metastasis. Cell Biophys 23: 3-89, 1993.

6. Kavic SM and Basson MD: Environmental factors of temperature, humidity, serum accumulation, and cell seeding increase colon cancer cell adhesion in vitro, with partial characterization of the serum component responsible for pressure-stimulated adhesion. J Surg Res 98: 89-96, 2001.

7. Wirtz D, Konstantopoulos K and Searson PC: The physics of cancer: The role of physical interactions and mechanical forces in metastasis. Nat Rev Cancer 11: 512-522, 2011.

8. Basson MD: An intracellular signal pathway that regulates cancer cell adhesion in response to extracellular forces. Cancer Res 68: 2-4, 2008.

9. Downey C, Alwan K, Thamilselvan V, Zhang L, Jiang Y, Rishi AK and Basson MD: Pressure stimulates breast cancer cell adhesion independently of cell cycle and apoptosis regulatory protein (CARP)-1 regulation of focal adhesion kinase. Am J Surg 192: 631-635, 2006.

10. Conway WC, Van der Voort van Zyp J, Thamilselvan V, Walsh MF, Crowe DL and Basson MD: Paxillin modulates squamous cancer cell adhesion and is important in pressure-augmented adhesion. $\mathrm{J}$ Cell Biochem 98: 1507-1516, 2006.

11. Perry BC, Wang S and Basson MD: Extracellular pressure stimulates adhesion of sarcoma cells via activation of focal adhesion kinase and Akt. Am J Surg 200: 610-614, 2010.

12. Basson MD, Yu CF, Herden-Kirchoff $O$, Ellermeier $M$, Sanders MA, Merrell RC and Sumpio BE: Effects of increased ambient pressure on colon cancer cell adhesion. J Cell Biochem 78: 47-61, 2000.

13. Daskalakis M, Scheffel O and Weiner RA: High flow insufflation for the maintenance of the pneumoperitoneum during bariatric surgery. Obes Facts 2 (Suppl 1): 37-40, 2009.

14. Thamilselvan V and Basson MD: The role of the cytoskeleton in differentially regulating pressure-mediated effects on malignant colonocyte focal adhesion signaling and cell adhesion. Carcinogenesis 26: 1687-1697, 2005.

15. Parsons JT: Focal adhesion kinase: The first ten years. J Cell Sci 116: 1409-1416, 2003.

16. Wang S and Basson MD: Integrin-linked kinase: A multi-functional regulator modulating extracellular pressure-stimulated cancer cell adhesion through focal adhesion kinase and AKT. Cell Oncol 31: 273-289, 2009.

17. Wang S and Basson MD: Akt directly regulates focal adhesion kinase through association and serine phosphorylation: Implication for pressure-induced colon cancer metastasis. Am J Physiol Cell Physiol 300: C657-C670, 2011.

18. Golubovskaya VM: Targeting FAK in human cancer: From finding to first clinical trials. Front Biosci 19: 687-706, 2014.

19. Lv PC, Jiang AQ, Zhang WM and Zhu HL: FAK inhibitors in Cancer, a patent review. Expert Opin Ther Pat 28: 139-145, 2018. 
20. Schultze A and Fiedler W: Therapeutic potential and limitations of new FAK inhibitors in the treatment of cancer. Expert Opin Investig Drugs 19: 777-788, 2010.

21. Sulzmaier FJ, Jean $C$ and Schlaepfer DD: FAK in cancer: Mechanistic findings and clinical applications. Nat Rev Cancer 14: 598-610, 2014.

22. Basson MD, Zeng B and Wang S: Akt1 binds focal adhesion kinase via the Akt1 kinase domain independently of the pleckstrin homology domain. J Physiol Pharmacol 66: 701-709, 2015.

23. Basson MD, Zeng B and Wang S: The C-terminal region of the focal adhesion kinase F1 domain binds Akt1 and inhibits pressure-induced cell adhesion. J Physiol Pharmacol 68: 375-383, 2017.

24. Zeng B, Devadoss D, Wang S, Vomhof-DeKrey EE, Kuhn LA and Basson MD: Inhibition of pressure-activated cancer cell adhesion by FAK-derived peptides. Oncotarget 8: 98051-98067, 2017.

25. Marqus S, Pirogova E and Piva TJ: Evaluation of the use of therapeutic peptides for cancer treatment. J Biomed Sci 24: 21, 2017.

26. Otvos L Jr and Wade JD: Current challenges in peptide-based drug discovery. Front Chem 2: 62, 2014.

27. Raschka S, More SK, Devadoss D, Zeng B, Kuhn LA and Basson MD: Identification of potential small-molecule protein-protein inhibitors of cancer metastasis by 3D epitope-based computational screening. J Physiol Pharmacol 69: doi: 10.26402/jpp.2018.2.11.

28. Thamilselvan V, Craig DH and Basson MD: FAK association with multiple signal proteins mediates pressure-induced colon cancer cell adhesion via a Src-dependent PI3K/Akt pathway. FASEB J 21: 1730-1741, 2007.

29. Allain F, Vanpouille C, Carpentier M, Slomianny MC, Durieux S and Spik G: Interaction with glycosaminoglycans is required for cyclophilin B to trigger integrin-mediated adhesion of peripheral blood T lymphocytes to extracellular matrix. Proc Natl Acad Sci USA 99: 2714-2719, 2002.

30. Canavan HE, Cheng X, Graham DJ, Ratner BD and Castner DG: Cell sheet detachment affects the extracellular matrix: A surface science study comparing thermal liftoff, enzymatic, and mechanical methods. J Biomed Mater Res A 75: 1-13, 2005.

31. Craig DH, Downey C and Basson MD: SiRNA-mediated reduction of alpha-actinin-1 inhibits pressure-induced murine tumor cell wound implantation and enhances tumor-free survival. Neoplasia 10: 217-222, 2008.

32. Claycombe KJ, Vomhof-DeKrey EE, Garcia R, Johnson WT, Uthus E and Roemmich JN: Decreased beige adipocyte number and mitochondrial respiration coincide with increased histone methyl transferase (G9a) and reduced FGF21 gene expression in Sprague-Dawley rats fed prenatal low protein and postnatal high-fat diets. J Nutr Biochem 31: 113-121, 2016.

33. Vomhof-DeKrey E, Darland D, Ghribi O, Bundy A, Roemmich J and Claycombe K: Maternal low protein diet leads to placental angiogenic compensation via dysregulated M1/M2 macrophages and TNFa expression in Sprague-Dawley rats. J Reprod Immunol 118: 9-17, 2016.

34. Velling T, Nilsson S, Stefansson A and Johansson S: beta1-Integrins induce phosphorylation of Akt on serine 473 independently of focal adhesion kinase and Src family kinases. EMBO Rep 5: 901-905, 2004.

35. Huveneers S and Danen EH: Adhesion signaling - crosstalk between integrins, Src and Rho. J Cell Sci 122: 1059-1069, 2009.

36. Hynes RO: Integrins: Bidirectional, allosteric signaling machines. Cell 110: 673-687, 2002.

37. Turecková J, Vojtechová M, Krausová M, Sloncová E and Korínek V: Focal adhesion kinase functions as an akt downstream target in migration of colorectal cancer cells. Transl Oncol 2: 281-290, 2009.

38. Xia H, Nho RS, Kahm J, Kleidon J and Henke CA: Focal adhesion kinase is upstream of phosphatidylinositol3-kinase/Akt in regulating fibroblast survival in response to contraction of type I collagen matrices via a beta 1 integrin viability signaling pathway. J Biol Chem 279: 33024-33034, 2004.

39. Zhao J and Guan JL: Signal transduction by focal adhesion kinase in cancer. Cancer Metastasis Rev 28: 35-49, 2009.

40. Gayer CP and Basson MD: The effects of mechanical forces on intestinal physiology and pathology. Cell Signal 21: 1237-1244, 2009.

41. Vleming LJ, Baelde JJ, Westendorp RG, Daha MR, van Es LA and Bruijn JA: Progression of chronic renal disease in humans is associated with the deposition of basement membrane components and decorin in the interstitial extracellular matrix. Clin Nephrol 44: 211-219, 1995.
42. Tomar A and Schlaepfer DD: A PAK-activated linker for EGFR and FAK. Dev Cell 18: 170-172, 2010.

43. Nagano M, Hoshino D, Koshikawa N, Akizawa T and Seiki M Turnover of focal adhesions and cancer cell migration. Int J Cell Biol 2012: 310616, 2012

44. Schlaepfer DD, Hauck CR and Sieg DJ: Signaling through focal adhesion kinase. Prog Biophys Mol Biol 71: 435-478, 1999.

45. Higuchi M, Kihara R, Okazaki T, Aoki I, Suetsugu S and Gotoh Y: Akt1 promotes focal adhesion disassembly and cell motility through phosphorylation of FAK in growth factor-stimulated cells. J Cell Sci 126: 745-755, 2013.

46. Zhao X and Guan JL: Focal adhesion kinase and its signaling pathways in cell migration and angiogenesis. Adv Drug Deliv Rev 63: 610-615, 2011

47. Manning BD and Toker A: AKT/PKB Signaling: Navigating the Network. Cell 169: 381-405, 2017.

48. O'toole A, Moule SK, Lockyer PJ and Halestrap AP: Tumour necrosis factor-alpha activation of protein kinase B in WEHI-164 cells is accompanied by increased phosphorylation of Ser473, but not Thr308. Biochem J 359: 119-127, 2001.

49. Kitagawa K, Takasawa K, Kuwabara K, Sasaki T, Tanaka S, Mabuchi T, Sugiura S, Omura-Matsuoka E, Matsumoto M and Hori M: Differential Akt phosphorylation at Ser473 and Thr308 in cultured neurons after exposure to glutamate in rats. Neurosci Lett 333: 187-190, 2002.

50. Salazar EP, Hunger-Glaser I and Rozengurt E: Dissociation of focal adhesion kinase and paxillin tyrosine phosphorylation induced by bombesin and lysophosphatidic acid from epidermal growth factor receptor transactivation in Swiss $3 \mathrm{~T} 3$ cells. J Cell Physiol 194: 314-324, 2003.

51. Mitra SK, Hanson DA and Schlaepfer DD: Focal adhesion kinase: In command and control of cell motility. Nat Rev Mol Cell Biol 6: 56-68, 2005.

52. Mitra SK and Schlaepfer DD: Integrin-regulated FAK-Src signaling in normal and cancer cells. Curr Opin Cell Biol 18: $516-523,2006$

53. Shiratsuchi $\mathrm{H}$ and Basson MD: Akt2, but not Akt1 or Akt3 mediates pressure-stimulated serum-opsonized latex bead phagocytosis through activating mTOR and p70 S6 kinase. J Cell Biochem 102: 353-367, 2007.

54. Thamilselvan V and Basson MD: Pressure activates colon cancer cell adhesion by inside-out focal adhesion complex and actin cytoskeletal signaling. Gastroenterology 126: 8-18, 2004.

55. van Zyp J, Conway WC, Craig DH, van Zyp N, Thamilselvan V and Basson MD: Extracellular pressure stimulates tumor cell adhesion in vitro by paxillin activation. Cancer Biol Ther 5: $1169-1178,2006$

56. Goreczny GJ, Ouderkirk-Pecone JL, Olson EC, Krendel M and Turner CE: Hic-5 remodeling of the stromal matrix promotes breast tumor progression. Oncogene 36: 2693-2703, 2017.

57. Lee BY, Hochgräfe F, Lin HM, Castillo L, Wu J, Raftery MJ, Martin Shreeve S, Horvath LG and Daly RJ: Phosphoproteomic profiling identifies focal adhesion kinase as a mediator of docetaxel resistance in castrate-resistant prostate cancer. Mol Cancer Ther 13: 190-201, 2014.

58. Srinivas V, Datta SA, Ramakrishna T and Rao CM: Studies on the alpha-crystallin target protein binding sites: Sequential binding with two target proteins. Mol Vis 7: 114-119, 2001.

59. Wang $\mathrm{H}$, Zhu Y, Zhao M, Wu C, Zhang P, Tang L, Zhang H, Chen X, Yang Y and Liu G: miRNA-29c suppresses lung cancer cell adhesion to extracellular matrix and metastasis by targeting integrin $\beta 1$ and matrix metalloproteinase2 (MMP2). PLoS One 8: e70192, 2013.

60. Nelson H, Sargent DJ, Wieand HS, Fleshman J, Anvari M, Stryker SJ, Beart RW Jr, Hellinger M, Flanagan R Jr, Peters W, et al; Clinical Outcomes of Surgical Therapy Study Group: A comparison of laparoscopically assisted and open colectomy for colon cancer. N Engl J Med 350: 2050-2059, 2004.

61. Fujita S, Kudo N, Akasu T and Moriya Y: Detection of cytokeratin 19 and 20 mRNA in peripheral and mesenteric blood from colorectal cancer patients and their prognosis. Int J Colorectal Dis 16: 141-146, 2001.

62. Guller U, Zajac P, Schnider A, Bösch B, Vorburger S, Zuber M, Spagnoli GC, Oertli D, Maurer R, Metzger U, et al: Disseminated single tumor cells as detected by real-time quantitative polymerase chain reaction represent a prognostic factor in patients undergoing surgery for colorectal cancer. Ann Surg 236: 768-775, discussion 775-776, 2002 
63. Ishida H, Hashimoto D, Takeuchi I, Yokoyama M, Okita T and Hoshino T: Liver metastases are less established after gasless laparoscopy than after carbon dioxide pneumoperitoneum and laparotomy in a mouse model. Surg Endosc 16: 193-196, 2002.

64. Lee SW, Whelan RL, Southall JC and Bessler M: Abdominal wound tumor recurrence after open and laparoscopic-assisted splenectomy in a murine model. Dis Colon Rectum 41: 824-831, 1998.

65. Melamed A, Margul DJ, Chen L, Keating NL, Del Carmen MG, Yang J, Seagle BL, Alexander A, Barber EL, Rice LW, et al: Survival after Minimally Invasive Radical Hysterectomy for Early-Stage Cervical Cancer. N Engl J Med 379: 1905-1914, 2018.
66. Ramirez PT, Frumovitz M, Pareja R, Lopez A, Vieira M, Ribeiro R, Buda A, Yan X, Shuzhong Y, Chetty N, et al: Minimally Invasive versus Abdominal Radical Hysterectomy for Cervical Cancer. N Engl J Med 379: 1895-1904, 2018.

67. Umpleby HC, Fermor B, Symes MO and Williamson RC: Viability of exfoliated colorectal carcinoma cells. Br J Surg 71: 659-663, 1984

68. Thamilselvan V, Patel A, van der Voort van Zyp J and Basson MD: Colon cancer cell adhesion in response to Src kinase activation and actin-cytoskeleton by non-laminar shear stress. J Cell Biochem 92: 361-371, 2004.

69. Sugarbaker PH: Successful management of microscopic residual disease in large bowel cancer. Cancer Chemother Pharmacol 43 (Suppl): S15-S25, 1999. 